\title{
DESENVOLVIMENTO DE UM INSTRUMENTO PARA AVALIAÇÃO DA CONDIÇÃO DE ESTRADAS NÃO PAVIMENTADAS
}

\author{
Device development for assessment of unpaved roads condition
}

\author{
Heliana Barbosa Fontenele ${ }^{1}$, José Leomar Fernandes Júnior ${ }^{2}$
}

Recebido em 17 de dezembro de 2012; recebido para revisão em 20 de dezembro de 2012; aceito em 30 de janeiro de 2013; disponível on-line em 21 de junho de 2013.

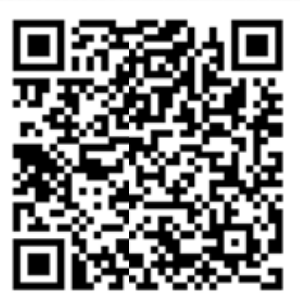

\section{PALAVRAS CHAVES:}

Estrada de terra;

Avaliação subjetiva de estradas;

Avaliação por Escala visual;

\section{KEYWORDS:}

Unpaved road;

Subjective rating of roads;

Rating by Visual scale;

\section{* Contato com os autores:}

${ }^{1}$ e-mail : heliana@uel.br (H. B. Fontenele)

Professora do Dep. de Construção Civil do Curso de Engenharia Civil da Universidade Estadual de Londrina (UEL)

2e-mail : leomar@sc.usp.br

(J. L. Fernandes Júnior)

Professor do Dep. de Transportes do Curso de Engenharia Civil da Escola de Engenharia de São Carlos - Umiversidade de São Paulo (USP) desenvolvimento socioeconômico de uma região, sendo responsáveis pelo escoamento agrícola e a integração entre o campo e a cidade através do acesso da comunidade rural aos serviços de saúde, educação e lazer. Infelizmente, essas estradas se deterioram mais rapidamente que as estradas pavimentadas, tornando necessária uma frequente manutenção que, geralmente, é feita de forma empírica e incorreta, implicando, por sua vez, em altas taxas de deterioração e, consequentemente, numa sequencia de custos aos usuários, que variam de acordo com as características da superfície. Tendo em vista o exposto, este trabalho tem como objetivo contribuir ao procedimento de avaliações subjetivas executadas em tais estradas com vistas à aplicação em um sistema de gerência, permitindo, dessa maneira, que as seções que precisam de melhorias sejam conhecidas e priorizadas. Utilizam-se, nesta pesquisa, escalas visuais, elaboradas com auxílio de especialistas, para medir as características referentes a conforto, conveniência e, principalmente, custos, tanto para os usuários como para os organismos públicos responsáveis pela manutenção e reabilitação da malha viária. Foram utilizadas fotografias de trechos de estradas de terra representativos de cada posição na escala de classificação adotada para a avaliação superficial e, após a elaboração da escala, foram formados dois painéis de avaliadores. A um deles foi fornecido um treinamento detalhado, utilizando a escala visual elaborada, e ao outro foram repassadas apenas informações básicas sobre o processo de avaliação. Ambos os painéis avaliaram fotogriafias de variadas condições de estradas e as classificaram para que fosse avaliada a influência exercida pelo treinamento no julgamento da qualidade superficial das estradas. Concluiu-se que ambas as formas de avaliação, com ou sem treinamento, podem ser utilizadas, pois a coerência oriunda da avaliação com ambos os procedimentos foram muito próximas.

ABSTRACT: The unpaved roads represent an important rolle in social and economical development of a region, being responsible for agricultural flow and the integration between countryside and city through rural community to health, education and recreation services. Unfortunately, these roads deteriorate faster than paved roads, being necessary a frequent maintenance that, usually, is dine in an incorrect and empirical way, causing elevated deterioration rates and consequently in a sequence of costs to the users, that varies according to superficial characteristics. In front of the exposed, this work has the objective to contribute to subjective ratings proceedings executed on such roads to aim the management of systems allowing that the sections that need improvement are known and prioritized. In this research they were used visual scales elaborated with aid of specialists to measure comfort, convenience and costs characteristics as much for the users as for the highway agencies responsible for management and rehabilitation of road net. Pictures of unpaved roads sections representing each position of subjective rating scale, and after the scale elaboration were formed two panels of raters. To one of them a detailed training was supplied using the visual scale and to the other panel were passed only basic information about the rating proces:s. Both panel rated pictures of diverse roads conditions and qualified them to evaluate the influence training on judgment of roads superficial quality. It was conclude that both the evaluation forms, with or without training, could be used, therefore the deriving coherence in both procedures was very adjacent.

RESUMO: As estradas não pavimentadas representam um importante papel no 


\section{INTRODUÇÃO}

As estradas não pavimentadas, também chamadas de estradas de terra, representam um importante papel no desenvolvimento socioeconômico de uma região, sendo responsáveis pelo escoamento agrícola e a integração entre o campo e a cidade através do acesso da comunidade rural aos serviços de saúde, educação e lazer. De acordo com Chamorro e Tighe (2011) tais estradas podem representar mais de $80 \%$ da rede viária de um país, assim, dada a sua importância socioeconômica ao bem-estar e desenvolvimento das populações rurais, os órgãos responsáveis pela sua gestão devem mantê-las em condições ideais para o tráfego.

A partir da característica que essas estradas possuem de perder o material de sua superfície pela ação combinada do tráfego e das intempéries percebe-se que elas se deterioram mais rapidamente que as estradas pavimentadas, tornando necessária uma frequente manutenção. Infelizmente, as atividades destinadas à conservação dessas vias são feitas, geralmente, de forma empírica e incorreta, implicando, por sua vez, em altas taxas de deterioração e, consequentemente, numa sequencia de custos aos usuários (custos operacionais dos veículos, custo com o tempo perdido no percurso, custos com o desconforto e custos de acidentes), que variam de acordo com a variação das características da superfície, dentre elas a irregularidade longitudinal do pavimento.

A partir do AASHO Road Test (experimento conduzido na pista experimental da American Association of State Highway Officials nos anos de 1958 a 1961) ficou reconhecido o papel do usuário na formulação de notas para representar a serventia do pavimento (Carey e Irick, 1960). Desde então, significativa importância tem sido dada à satisfação do usuário quanto à qualidade de viagem proporcionada pela via como auxílio à gerência, permitindo, dessa maneira, que as seções que precisam de melhorias sejam conhecidas e priorizadas.

Em decorrência da subjetividade envolvida no processo de avaliação do pavimento, certo número de fatores pode distorcer o julgamento dos usuários a vários graus de classificação, tornando a medição da qualidade de viagem um pouco mais difícil. Uma das alternativas para evitar tais distorções e, consequentemente, um comprometimento dos resultados, é a definição de um adequado planejamento do procedimento em campo, indo desde a escolha dos usuários e seu treinamento à escolha dos segmentos a serem avaliados. Dessa forma, este trabalho tem como objetivo verificar a influência do treinamento do painel de avaliadores, utilizando para isso uma escala visual elaborada para o estudo.

\section{AS ESTRADAS NÃO PAVIMENTADAS}

\subsection{CARACTERÍSTICAS}

As estradas não pavimentadas são caracterizadas, de acordo com Dobson e Postill (1983), como estradas de baixo volume por apresentarem tráfego diário médio de 0 a 400 veículos. Segundo Jones e Ksaibati (2012), as características do tráfego em tais estradas diferem daquelas em estradas pavimentadas devido a vários fatores, como: diferentes padrões de projeto; níveis de manutenção; tipo de motoristas e características de segurança viária. A sua constituição é geralmente de solo local, com ou sem adição de agregados, e sem nenhum tipo de revestimento asfáltico ou de concreto.

Suas larguras variam muito e são modificadas de acordo com a necessidade local. Dobson e Postill (1983) também apontam que os limites de velocidade nessas estradas não são estabelecidos, assim a maioria dos usuários utiliza, de acordo com as condições da superfície e de visibilidade da estrada, os limites comuns às estradas pavimentadas.

Essas estradas, que possuem um tempo de duração de cerca de $10 \%$ do tempo de vida das estradas pavimentadas, representam grande parte da rede rodoviária dos países e, geralmente, estão localizadas nas áreas rurais. De acordo com dados do GEIPOT (2008), considerando todas as regiões do país, no ano de 2000 tais estradas representavam aproximadamente $90,5 \%$ da malha rodoviária brasileira; dessa porcentagem, $28 \%$ estão localizadas na região Sul, sendo que uma porcentagem próxima a $16 \%$ pertencem somente ao Estado do Paraná (considerando as vias rurais de todas as esferas: federais; estaduais e municipais). De acordo com Namur e Solminihac (2009) nos países em desenvolvimento (leia-se países emergentes) a maioria das estradas ainda é do tipo não pavimentada.

\subsection{MANUTENÇÃO}

A manutenção de estradas visa diminuir a deterioração da via prolongando sua vida útil e reduzir os prejuízos decorrentes da má condição da estrada; prejuízos estes que envolvem não só os custos operacionais dos veículos, mas o prejuízo social devido à interrupção do transporte entre campo e cidade que 
conduzem crianças e professores às escolas, pacientes aos hospitais e tantos outros.

De acordo com Huntington e Ksaibati (2011) os órgãos responsáveis pela manutenção agem para resolver os problemas na via de uma entre duas formas: respondendo somente a reclamações ou sem levar em consideração questões econômicas ou de engenharia; ambas as maneiras resultam em manutenções ineficientes que só aumentam o custo do processo e reduzem o valor dos serviços prestados ao público.

Assim, a manutenção das estradas municipais não pavimentadas no Brasil é geralmente limitada às práticas emergenciais (recomposição do corpo estradal) e à manutenção corretiva de rotina (limpeza de valetas e sarjetas, recomposição dos dispositivos de drenagem, reconformação da plataforma). A manutenção preventiva periódica (melhoria do traçado, proteção à erosão, obras de drenagem, etc.) só é realizada em casos excepcionais.

\section{A AVALIAÇÃO DA SUPERFÍCIE DE ESTRADAS}

Em função do baixo volume de tráfego existente nas estradas não pavimentadas, de acordo com Chamorro et al. (2009), os recursos disponíveis para uma apropriada avaliação e manutenção são muito limitados, fazendo com que a avaliação da sua condição seja executada, geralmente, por métodos subjetivos, o que requer curto período de tempo e consequentemente poucos recursos. Ainda segundo tais autores, essa metodologia apresenta uma carência de objetividade no momento de quantificar o nível de defeitos, bem como quando se combinam os defeitos para atribuir a taxa de condição da superfície da estrada. Tal afirmação, segundo os autores, suscita a necessidade de aplicações de métodos objetivos para a avaliação de vias não pavimentadas. A seguir são abordados, de maneira sucinta, os tipos de avaliação que podem ser conduzidas para a avaliação da condição da superfície de estradas.

\subsection{AVALIAÇÃo SUBJETIVA}

A avaliação subjetiva determina a condição da superfície de um pavimento segundo o ponto de vista dos usuários. No Brasil, a avaliação subjetiva de pavimentos flexíveis é normalizada pelo DNIT (Departamento Nacional de Infraestrutura de Transportes) em sua norma PRO 009/2003, na qual sugere o uso do índice chamado de Valor de Serventia Atual (VSA) para caracterizar a condição do pavimento.

Durante a avaliação, o VSA é obtido através da média de notas atribuídas ao pavimento por membros de um painel de avaliação destinados a representar a opinião dos usuários de uma rodovia, tendo em mente a seguinte pergunta: "Como esta estrada me serviria se eu estivesse dirigindo meu carro sobre estradas como esta ao longo de todo o dia?".

Em tal procedimento, os avaliadores, devidamente treinados, percorrem os trechos das estradas na velocidade operacional neles permitida e, de dentro do veículo, atribuem notas subjetivas numa escala de zero a cinco pontos relacionados à qualidade do pavimento.

No decorrer da avaliação, de acordo com DNIT (2003a), não deverá haver nenhum tipo de comentário entre os avaliadores, somente deve ser levado em consideração o estado atual da superfície e não devem ser considerados: o aspecto do projeto geométrico; a resistência à derrapagem do revestimento; os cruzamentos rodoviários; as irregularidades nos acessos das pontes e aquelas ocasionais devidas a recalques de bueiros, mas sim, os buracos, saliências, irregularidades transversais e longitudinais da superfície. A avaliação deve ser realizada sob condições climáticas favoráveis.

É importante comentar que no estudo feito por Fontenele e Sória (2005) foi realizada uma análise de avaliações subjetivas feitas em estradas de terra por um painel de sete avaliadores, sendo verificadas algumas diferenças entre as avaliações de cada avaliador. Segundo conclusões do estudo estas já eram esperadas e perfeitamente compreensíveis, visto que um avaliador tende a avaliar melhor que outros, mas desde que dentro de certo limite de variação. Notou-se também que alguns membros se distanciaram da avaliação média do restante do grupo, mas isso não é indicativo que a melhor solução seria retirá-los do painel, para tanto seria necessário um estudo mais aprofundado para verificar a validade de suas inclusões no grupo. Além disso, as dispersões entre os avaliadores foram atribuídas ao fato do grande número de intervalos da escala de classificação e à inexperiência dos membros do painel, como também à fadiga dos avaliadores, pois as avaliações foram feitas durante todo o dia. Os autores ainda afirmaram que o uso de um painel de avaliadores, desde que treinados, pode ser utilizado para avaliação de trechos de estradas de terra. As avaliações subjetivas do painel foram consideradas, neste estudo, como válidas para representar as condições de trechos de uma estrada não pavimentada de 30 a $300 \mathrm{~m}$ de comprimento.

\subsubsection{Os aspectos psicológicos envolvidos na avaliação subjetiva}

A formação do juízo de cada avaliador está ligada a certos fatores de ordem externa que os 
influenciam psicologicamente na atribuição de suas impressões.

O avaliador passa por todo um processo durante seu julgamento. Esse processo, segundo Nair e Hudson (1986), envolve a interação entre veículo, perfil longitudinal da superfície do pavimento e próprio avaliador. Essa interação dinâmica é responsável pelo envio de um estímulo físico ao usuário (vibrações transmitidas pelo veículo ao se movimentar pelo pavimento) e a geração de contínuas sensações. Assim, nem sempre quando o mesmo estímulo é enviado ao avaliador em diferentes condições esse produz a mesma sensação, é justamente neste ponto que se percebe nitidamente a subjetividade do processo.

Nair e Hudson (1986) afirmam que o julgamento começa quando estímulos psicológicos são recebidos pelo avaliador a partir das ações do experimentador, de suas instruções e dos estímulos provenientes do objeto avaliado. Neste ponto o avaliador então reage formando o juízo (notas) sobre a qualidade do pavimento.

\subsubsection{Planejando a avaliação subjetiva}

Em função dos aspectos relatados anteriormente, se faz necessário um planejamento do processo de avaliação subjetiva para que distorções sejam evitadas e assim não comprometam o resultado final da classificação da condição das estradas. A seguir são abordados os itens a serem considerados no planejamento.

\section{a. Determinação do painel de avaliação}

Na determinação do painel, Pereira e Delanne (1999) apontam que basicamente dois pontos devem ser observados: seu tamanho e sua composição; tendo em vista a redução de prováveis erros na classificação subjetiva das estradas.

Nos estudos de Nakamura e Michael (1963), Pereira e Delanne (1999) e Fontenele (2001), a opinião da formação de um painel com poucos avaliadores é defendida. A partir desses trabalhos a utilização de painéis com 3 ou no máximo 10 avaliadores é considerada suficiente para obter resultados confiáveis.

Quanto à composição, grande importância deve ser dada à escolha dos membros do painel de avaliação, afinal eles serão responsáveis pela representação da opinião de todos os usuários daquela estrada. De acordo com Carey e Irick (1960) e Weaver e Clark (1977), os indivíduos devem ser selecionados de vários segmentos de usuários com opiniões e atitudes divergentes, levando em consideração a habilidade de ser sincero, fazer julgamentos independentes e seguir instruções simples.

\section{b. Treinamento do painel}

O objetivo do treinamento é definir, clara e precisamente, os aspectos envolvidos na avaliação para que os avaliadores fiquem cientes das regras e definições da avaliação antes de partirem definitivamente ao campo. Janoff e Nick (1985) afirmam que o treinamento deve consistir das seguintes explanações:

- a característica da qualidade de viagem sendo avaliada;

- a forma de utilização da escala que será empregada;

- o processo de avaliação, incluindo as respostas às questões relativas a este processo;

- a confidencialidade das avaliações;

- as definições que os avaliadores devem compreender;

- qualquer questão que os avaliadores apresentem relativas às suas atividades, aos procedimentos, definições etc.

As diretrizes devem ser dadas aos avaliadores no início de cada período de avaliação a partir de um manual de instruções para remover o efeito de interpretação pessoal dos experimentadores. As questões provenientes durante o estudo devem ser esclarecidas pelo experimentador condutor da pesquisa sempre a todos os avaliadores uniformemente.

\section{c. Escolha dos segmentos}

Um tamanho mínimo de segmento de estudo deve ser escolhido que permita que o avaliador tenha tempo suficiente para adquirir uma percepção estabilizada do estímulo transmitido a partir da superfície do pavimento. Quando a extensão é muito elevada, de acordo com Weaver e Clark (1977), o avaliador corre o risco de "esquecer" o estímulo inicial, levando em consideração apenas o estímulo percebido nos últimos metros do segmento avaliado.

Além disso, ainda segundo Weaver e Clark (1977), as características físicas dos segmentos utilizados na avaliação devem ser analisadas com cuidado para que não influenciem no resultado final da avaliação do painel. Esses segmentos devem ser relativamente homogêneos e não devem conter características muito diferentes dos metros que o antecedem para que sua avaliação não seja influenciada.

\subsection{AVALIAÇÃO OBJETIVA}

Este tipo de avaliação sustenta-se em dados coletados a respeito dos defeitos existentes na seção da estrada a ser analisada (Haas et al., 1994). A avaliação pode ser executada de duas maneiras: (1) caminhadas 
ao longo da seção ou (2) num veículo em movimento. Para o levantamento de defeitos é necessário que os tipos de defeitos a serem considerados sejam selecionados e cuidadosas definições da aparência de cada um sejam estabelecidas. As definições devem estar escritas e acompanhadas por fotografias ou figuras que ajudem na sua identificação, incluindo sustentações para avaliar a severidade e medir sua extensão.

O DNIT (2003b) sugere dois procedimentos para a avaliação objetiva das condições de superfícies pavimentadas. O primeiro deles é uma avaliação baseada num índice que expressa o estado geral de um pavimento em função da incidência de defeitos, o Índice de Gravidade Global - IGG (DNIT, 2003b). O segundo procedimento, segundo DNER (1983), considera dados levantados sobre defeitos em segmentos testemunhas julgados homogêneos, permitindo, deste modo, calcular a percentagem da área afetada por cada tipo de defeito levantado.

Especificamente para avaliar a condição de estradas de terra, o método do Unpaved Road Condition Index (URCI) foi desenvolvido pelo U. S. Army Corps of Engineers e usa curvas de valores dedução para cada tipo e severidade do defeito presente no trecho analisado (Eaton, Gerard e Datillo, 1987). No Brasil, Fontenele (2001) e Sória e Fontenele (2003) apresentaram um estudo referente a adaptação de tal método para as condições brasileiras.

No Chile, em um trabalho mais atual, Chamorro et al. (2009) desenvolveram uma metodologia para avaliar as condições de estradas não pavimentadas com base em medidas objetivas dos defeitos superficiais, drenagem e nas características do perfil. Como resultado final do estudo equações que representavam o Índice de Condição de Estradas Não Pavimentadas - Unpaved Condition Index (UPCI) e os limites de condições foram desenvolvidos.

\section{ESCALAS DE AVALIAÇÃO DA CONDIÇÃO SUPERFICIAL DO PAVIMENTO}

Através do estudo conhecido como AASHO Road Test, segundo Carey e Irick (1960), ficou evidenciado que a serventia do pavimento era quantificável, sendo, além disso, apresentado que a serventia nada mais é que uma grandeza ou experiência psicológica e não apenas uma medida física derivada da irregularidade do pavimento.

Embora a subjetividade possa vir acompanhada de fatores que venham a distorcer o julgamento dos usuários, a serventia do pavimento ainda é perfeitamente quantificável. Com o intuito de minimizar as distorções dos julgamentos a maioria das investigações psicofísicas procura estabelecer escalas de medição da experiência psicológica e relatar esta a uma escala de medição do estímulo físico (HUTCHINSON, 1964).

Ainda tendo em mente as tendências e fatores que podem deturpar as avaliações dos usuários, Hartgen et al. (1982), preocupados com as condições e manutenção das vias pavimentadas da cidade de Nova York, utilizaram procedimentos para desenvolver escalas visuais para a avaliação da condição dos pavimentos. Assim, tendo em vista facilitar a avaliação da superfície das vias em campo, foram elaboradas escalas visuais de classificação, criando assim uma espécie de "guia", contendo nele imagens das mais variadas condições da superfície das vias e suas respectivas classificações.

O estudo conduzido em Nova York contou com a revisão de mais de 50 fotografias de pavimentos por oito especialistas na área. Tais fotografias foram avaliadas numa escala de 0 (zero) a 10 (dez) no tocante às condições de base e superfície de rolamento. Após esta fase, análises posteriores produziram um conjunto de fotografias representativas de cada posição na escala de valores.

Dessa forma, um painel de avaliadores foi treinado, com base na escala visual desenvolvida, para que posteriormente prosseguissem as avaliações subjetivas, em campo, das vias. Com esse método Hartgen et al. (1982) conseguiram realizar avaliações periódicas das condições das vias da cidade e aplicar os investimentos na área de forma mais rápida e eficaz.

\section{O EXPERIMENTO}

A partir do conhecimento de que na área de transportes escalas visuais e verbais têm sido usadas por muitos anos, como por exemplo, escalas verbais para medir as características dos serviços de transportes referentes a conforto, conveniência e custo, procurou-se construir neste trabalho uma escala visual para a avaliação superficial de estradas não pavimentadas.

Para a montagem da escala visual fotografias de estradas de terra em diversas condições superficiais foram feitas e posteriormente submetidas à classificação de um grupo de indivíduos na região de estudo com experiência na área de solos e estradas.

Após uma detalhada revisão, sessenta fotografias, contendo diversas condições superficiais, foram selecionadas para serem utilizadas na avaliação do grupo de especialistas. A partir de então, o grupo de 
especialistas classificou as imagens quanto à condição da sua superfície dentro de uma escala que variava de 0 (péssima) a 100 (excelente) pontos. Juntamente à classificação foi solicitado ao grupo que fizesse uma breve descrição sobre a provável velocidade, vibração e conforto de viagem oferecido pela superfície da via da fotografia em questão. Na atribuição de sua classificação, cada um dos especialistas levou em consideração os defeitos existentes na superfície da estrada (os buracos, saliências, irregularidades transversais e longitudinais da superfície) e o efeito negativo que estes provavelmente provocariam na qualidade e conforto de viagem sobre tal superfície.

A partir das avaliações dos especialistas, levando em consideração a consistência na avaliação, menor variância de avaliação, bem como aquelas que apresentavam os problemas da superfície de forma bastante clara, foram selecionadas fotos finais que melhor representavam as categorias da escala de avaliação adotada.

Desta forma, foi então montada uma escala visual de imagens relativas às condições das estradas não pavimentadas tendo-se duas fotografias para cada intervalo da escala de avaliação considerada no estudo. Aos intervalos de classificação foram aliadas informações quanto a possível velocidade a ser desenvolvida, bem como a qualidade de viagem, a vibração e conforto no trecho sob aquela condição.

\section{RESULTADOS E DISCUSSÃO}

\subsection{AVALIAÇÃo DAS FOTOGRAFIAS PELOS ESPECIALISTAS}

Neste item apresentam-se os resultados referentes à montagem da escala visual. A partir do gráfico da Figura 1 pode-se observar a correlação existente entre as notas fornecidas às sessenta fotografias utilizadas para a montagem final da escala visual da condição superficial de estradas não pavimentadas.

A partir do gráfico da Figura 1 verifica-se um coeficiente de determinação entre as avaliações individuais e do grupo, representada pela média, de cerca de 0,75 , ou seja, uma correlação entre os dados de 0,87 (87\%). Tal fato corrobora a coerência na avaliação dos especialistas tornando-a passível de ser utilizada na montagem da escala visual.

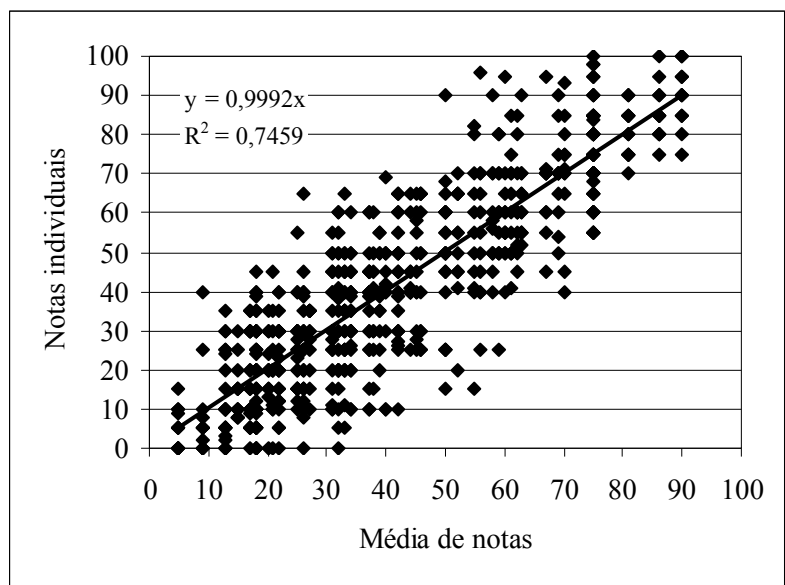

Figura 1: Gráfico de correlação entre as notas dos especialistas para montagem da escala visual.

\subsection{A ESCALA VISUAL}

A partir das avaliações que apresentavam menor variância, menor desvio padrão e boa clareza visual da condição da estrada, foi montada a escala visual relacionada aos intervalos da escala de valores que varia de 0 (zero) a 100 (cem) pontos. Nas Figuras 2 a 4 a escala visual desenvolvida está apresentada. Por fim, também foram adicionadas às imagens da escala visual características relacionadas ao conforto, velocidade desenvolvida, qualidade e trepidação ao longo do rolamento naquela superfície. Essas características estão relacionadas no Quadro 1.

\subsection{FORMAÇÃO DOS PAINÉIS DE AVALIAÇÃO}

Para o início do procedimento experimental foram formados dois painéis de avaliação. A um dos painéis foram oferecidas apenas instruções de como o processo de avaliação deveria ser feito e ao outro, além dessas instruções, foi dado um treinamento detalhado a partir da escala visual montada. O painel treinado foi formado por 9 indivíduos e o não treinado por 12.

$\mathrm{Na}$ etapa de instrução básica dos indivíduos "não treinados" teve-se como objetivo transmitir de forma clara instruções do que deve ser classificado e as condições necessárias para tal (deve ser considerada apenas a superfície da estrada, não deve haver distração durante a avaliação, não deve haver comentários de classificações com o pesquisador ou outros membros do painel, não há classificações certas ou erradas etc.), além de esclarecer as dúvidas por ventura existentes.

Já no treinamento do outro painel (painel treinado) a escala visual da condição da superfície de estradas não pavimentadas foi apresentada como 
referência. Esse processo teve a finalidade de nivelar os membros do painel do que deve ser considerado excelente e péssimo. Após a apresentação e discussão da escala visual elaborada neste trabalho, novas fotografias de estradas de terra em diferentes estágios de condição foram apresentadas ao grupo para que fossem classificadas com base no que foi exposto anteriormente. Um debate sobre a classificação de cada uma delas foi realizado até que a convergência de avaliações fosse razoável. Na Figura 5 apresenta-se a correlação obtida logo após o treinamento.

\begin{tabular}{|c|c|l|l|}
\hline \multicolumn{3}{|c|}{ Quadro 1: Características da superfície relacionadas aos intervalos da escala de classificação. } \\
\hline Nota & Classificação & \multicolumn{1}{c|}{ Características } \\
\hline $100-85$ & Excelente & $\begin{array}{l}\text { Alta qualidade de viagem; Pequena/nenhuma redução da velocidade; Velocidades: } \\
80 \mathrm{~km} / \mathrm{h} \text { ou mais; Pouca vibração do veículo e Pequeno/nenhum desconforto na } \\
\text { viagem. }\end{array}$ \\
\hline $85-70$ & Muito Boa & $\begin{array}{l}\text { Alta qualidade de viagem; Pequena/nenhuma redução da velocidade; Velocidades de } \\
70 \text { a } 80 \text { km/h; Pouca/média vibração do veículo e Pequeno/nenhum desconforto na } \\
\text { viagem. }\end{array}$ \\
\hline $70-55$ & Boa & $\begin{array}{l}\text { Média qualidade de viagem; Média redução da velocidade; Velocidades: de } 60 \text { a } 70 \\
\mathrm{~km} / \mathrm{h} ; \text { Pouca a média vibração do veículo e Médio desconforto na viagem. }\end{array}$ \\
\hline $55-40$ & Regular & $\begin{array}{l}\text { Média qualidade de viagem; Média redução da velocidade; Velocidades: de } 40 \text { a } 60 \\
\mathrm{~km} / \mathrm{h} ; \text { Média vibração do veículo e Médio desconforto na viagem. }\end{array}$ \\
\hline $40-25$ & Ruim & $\begin{array}{l}\text { Baixa qualidade de viagem; Grande redução da velocidade; Velocidades: de } 30 \text { a } 40 \\
\mathrm{~km} / \mathrm{h} ; \text { Muita vibração do veículo e Grande desconforto na viagem. }\end{array}$ \\
\hline $25-10$ & Muito Ruim & $\begin{array}{l}\text { Baixa qualidade de viagem; Grande redução da velocidade; Velocidades: de } 10 \text { a } 30 \\
\mathrm{~km} / \mathrm{h} ; \text { Muita vibração do veículo e Grande desconforto na viagem. }\end{array}$ \\
\hline $10-0$ & Péssima & $\begin{array}{l}\text { Baixa qualidade de viagem; Grande redução da velocidade; Velocidades: máximo } 10 \\
\mathrm{~km} / \mathrm{h} ; \text { Muita vibração do veículo e Grande desconforto na viagem. }\end{array}$ \\
\hline
\end{tabular}

Estrada Excelente

Nota: 90 Velocidade: $80 \mathrm{~km} / \mathrm{h}$

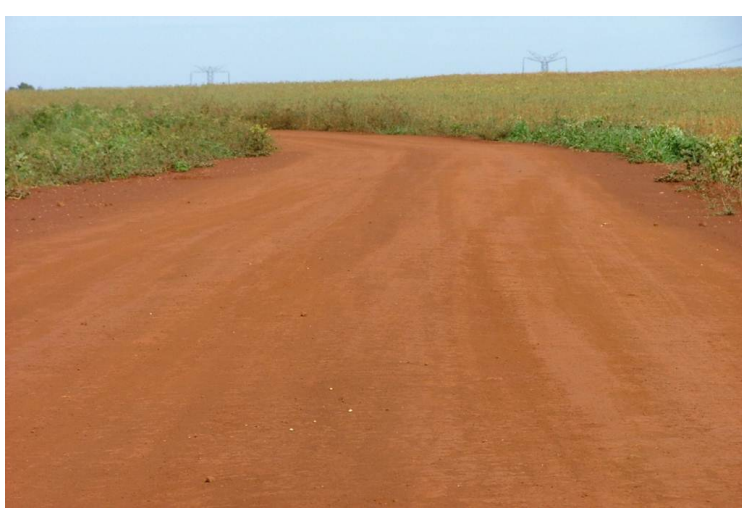

Estrada Muito Boa

Nota: 81 Velocidade: $75 \mathrm{~km} / \mathrm{h}$

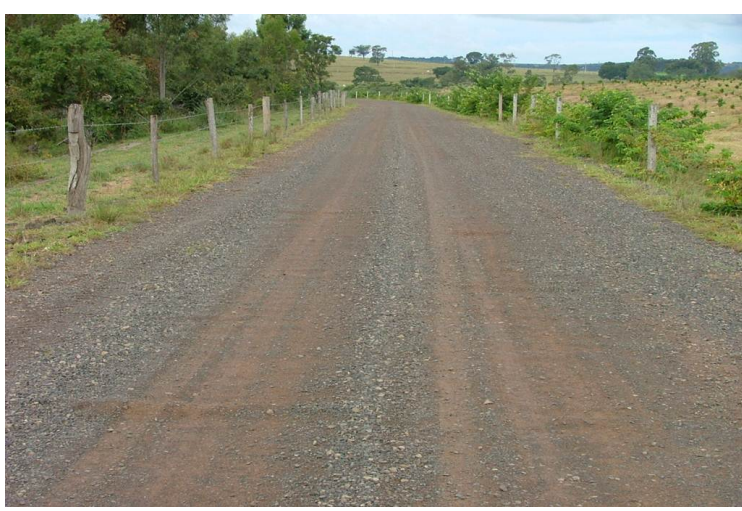

Estrada Excelente

Nota: 86 Velocidade: $80 \mathrm{~km} / \mathrm{h}$

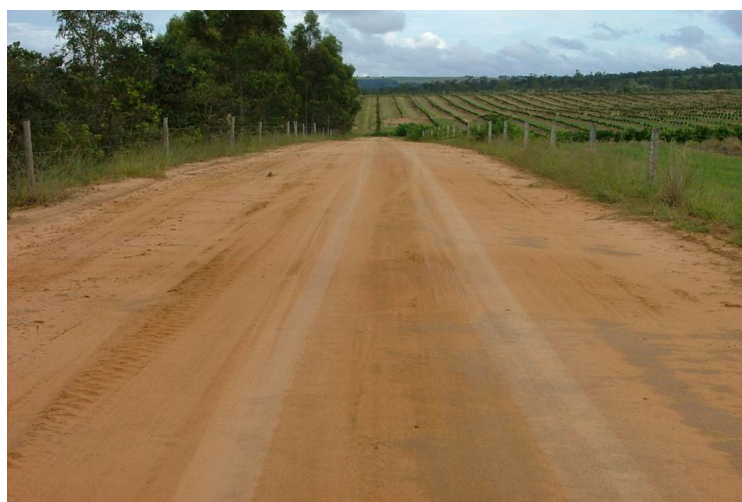

Estrada Muito Boa

Nota: 75 Velocidade: $70 \mathrm{~km} / \mathrm{h}$

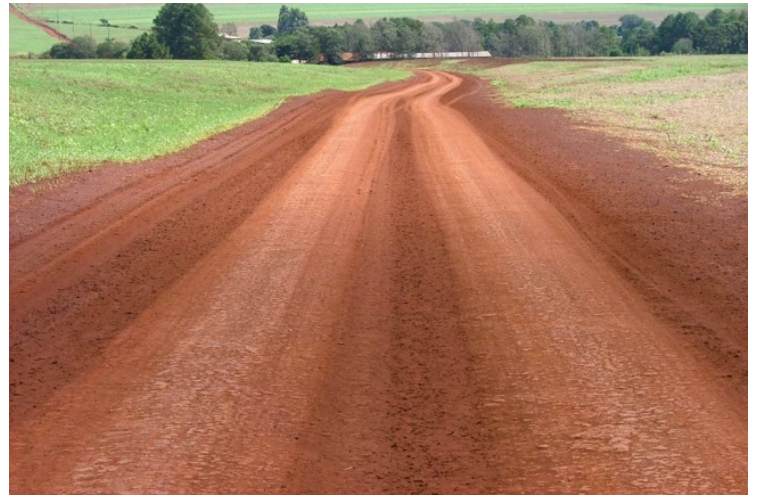

Figura 2: Escala visual: conceitos excelente e muito boa. 


\section{Estrada Boa}

Nota: 69 Velocidade: $70 \mathrm{~km} / \mathrm{h}$

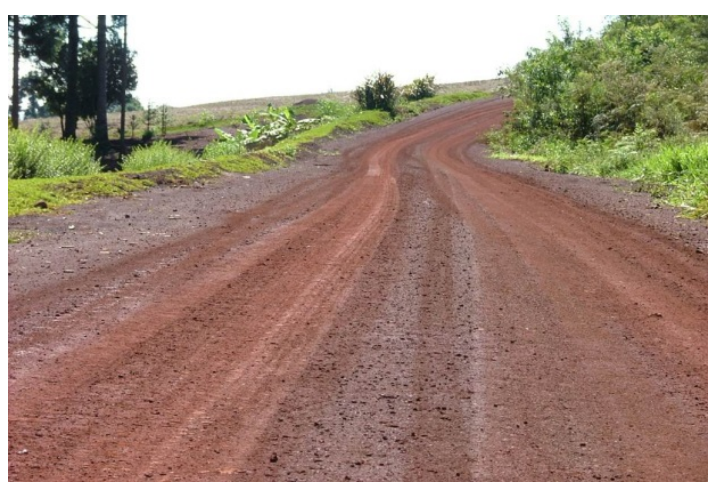

Estrada Regular

Nota: 52 Velocidade: $60 \mathrm{~km} / \mathrm{h}$

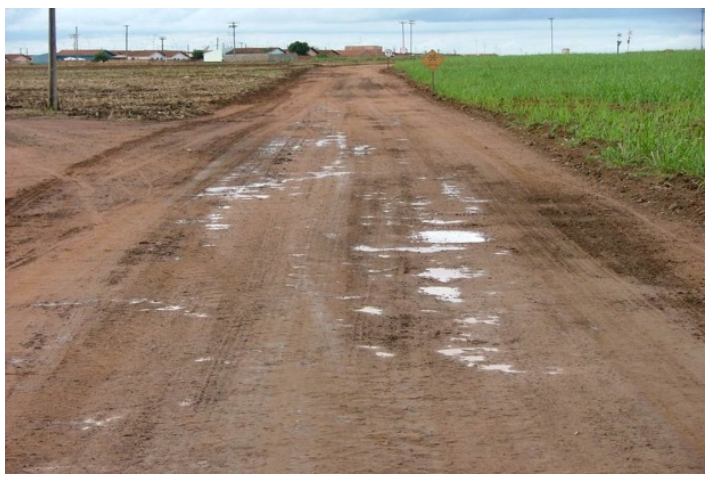

Estrada Ruim

Nota: 39 Velocidade: $40 \mathrm{~km} / \mathrm{h}$

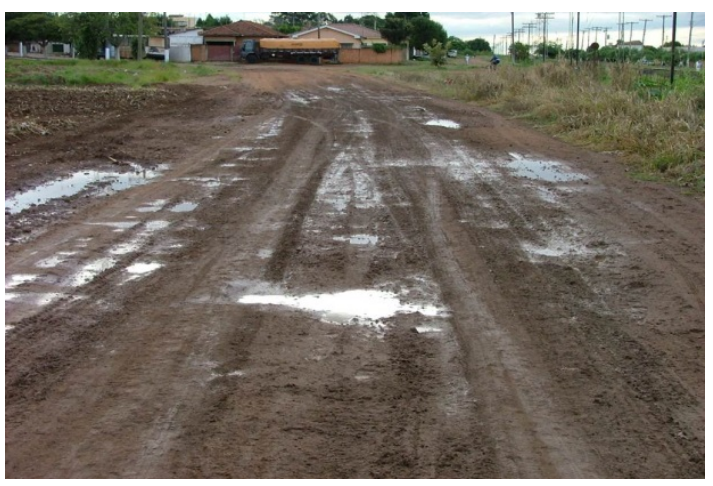

Estrada Muito Ruim

Nota: 20 Velocidade: $30 \mathrm{~km} / \mathrm{h}$

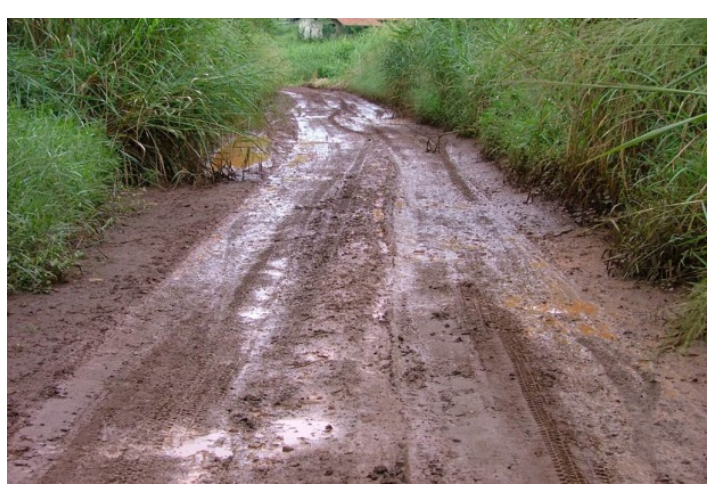

\section{Estrada Boa}

Nota: 59 Velocidade: $60 \mathrm{~km} / \mathrm{h}$

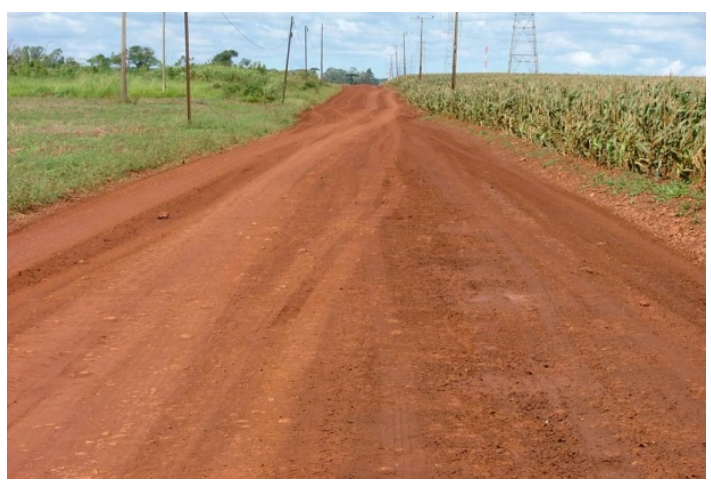

Estrada Regular

Nota: 44 Velocidade: $40 \mathrm{~km} / \mathrm{h}$

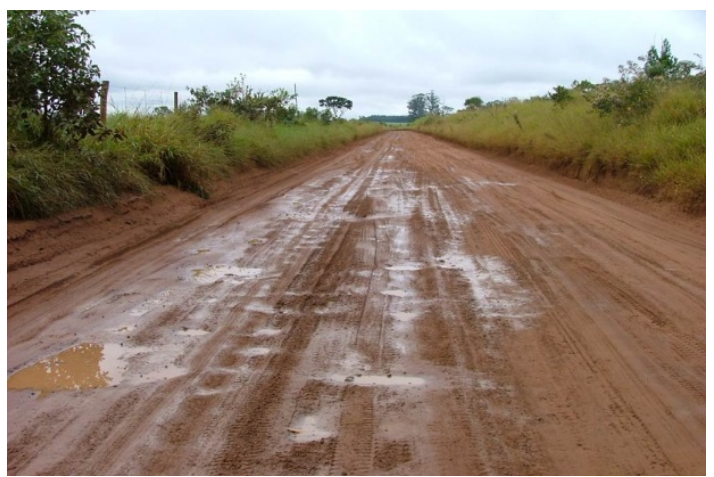

Estrada Ruim

Nota: 27 Velocidade: $30 \mathrm{~km} / \mathrm{h}$

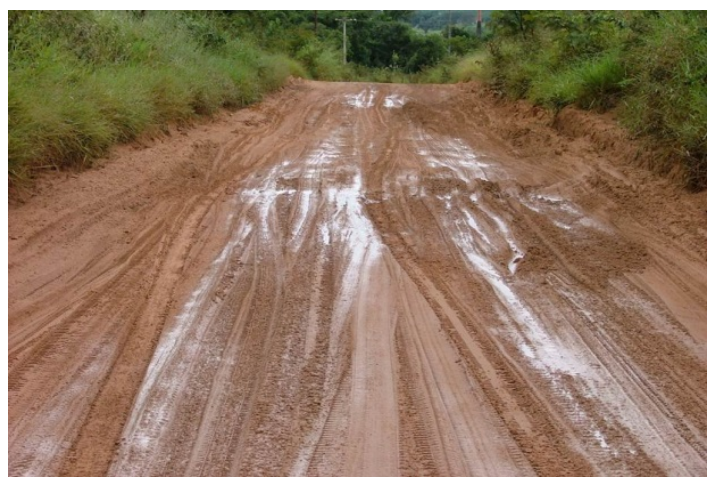

Estrada Muito Ruim

Nota: 15 Velocidade: $15 \mathrm{~km} / \mathrm{h}$

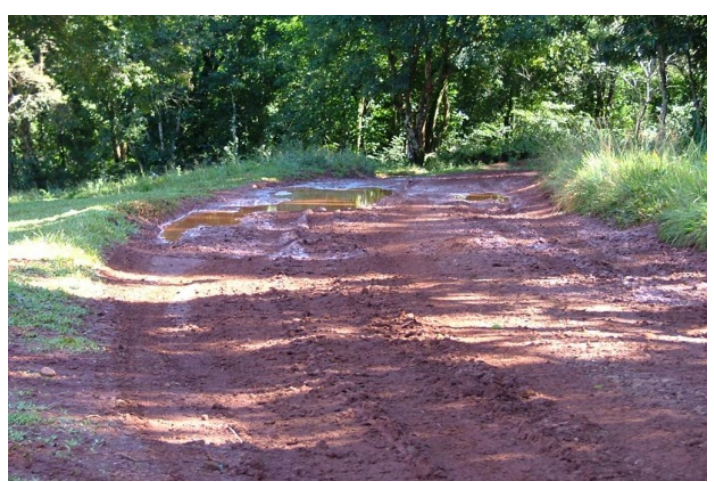

Figura 3: Escala visual: conceitos bom a muito ruim. 
Estrada Péssima

Nota: 9 Velocidade: $10 \mathrm{~km} / \mathrm{h}$

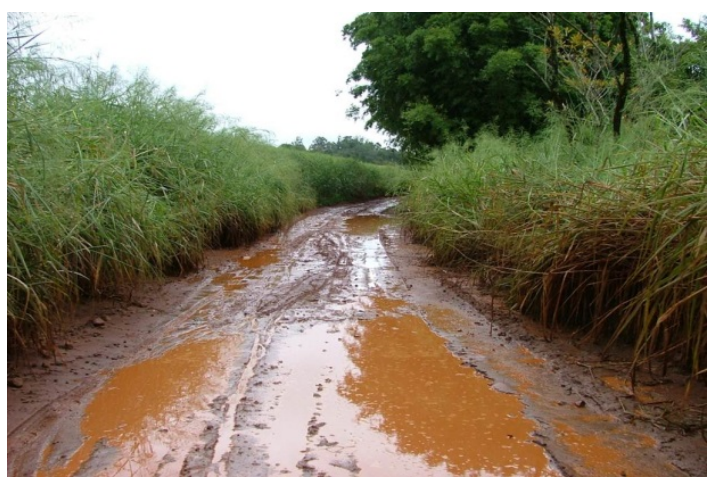

Estrada Péssima

Nota: 5 Velocidade: $10 \mathrm{~km} / \mathrm{h}$

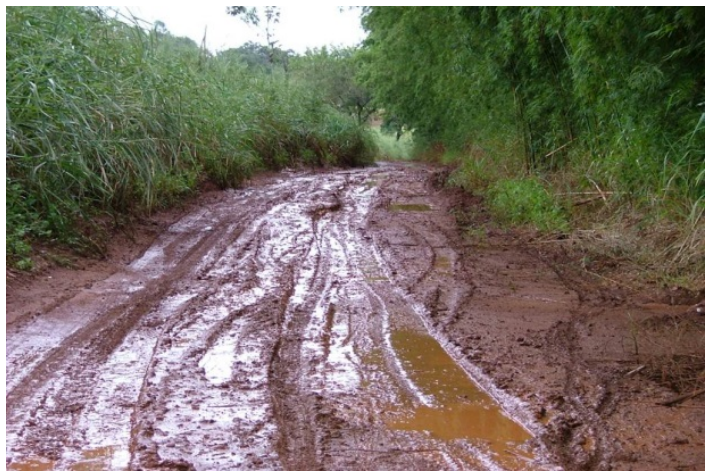

Figura 4: Escala visual: conceito péssimo.

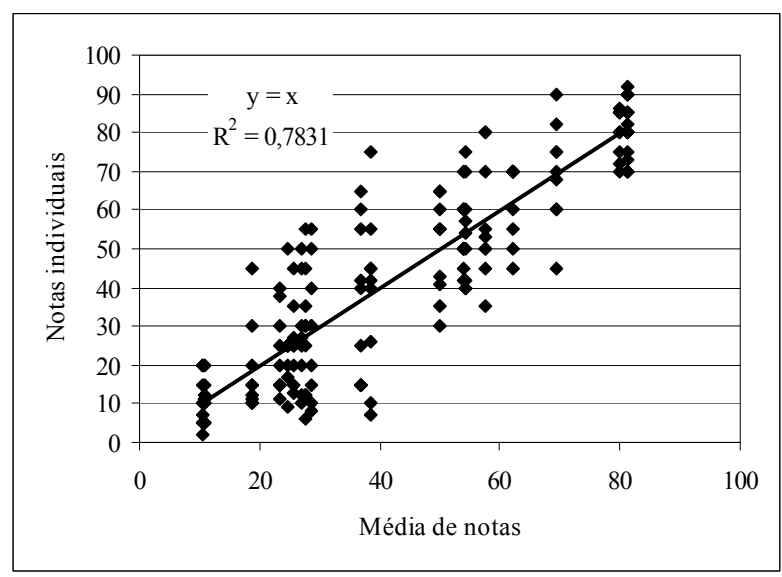

Figura 5: Gráfico de Correlação entre as notas do painel recém-treinado.

A partir da observação deste gráfico nota-se que a correlação alcançada no treinamento foi de cerca de $88 \%$, ou seja, $R=0,88$. Tal fato representa a coerência na atribuição das notas pelo painel treinado deixando a conclusão que o objetivo deste treinamento foi alcançado: nivelar os membros do painel do que deve ser considerado excelente e péssimo

Sendo assim, em escritório, foram apresentadas aos painéis, separadamente, 55 novas fotografias de estradas que estavam numeradas. A cada membro foi fornecido um formulário individual onde deveriam ser anotados o nome do avaliador, o número da fotografia e a nota atribuída à superfície daquela Estrada.

Com o intuito de verificar o papel do treinamento em um painel de avaliação de estradas as notas obtidas através do procedimento acima descrito foram correlacionadas obtendo-se os gráficos das Figuras 6 e 7.

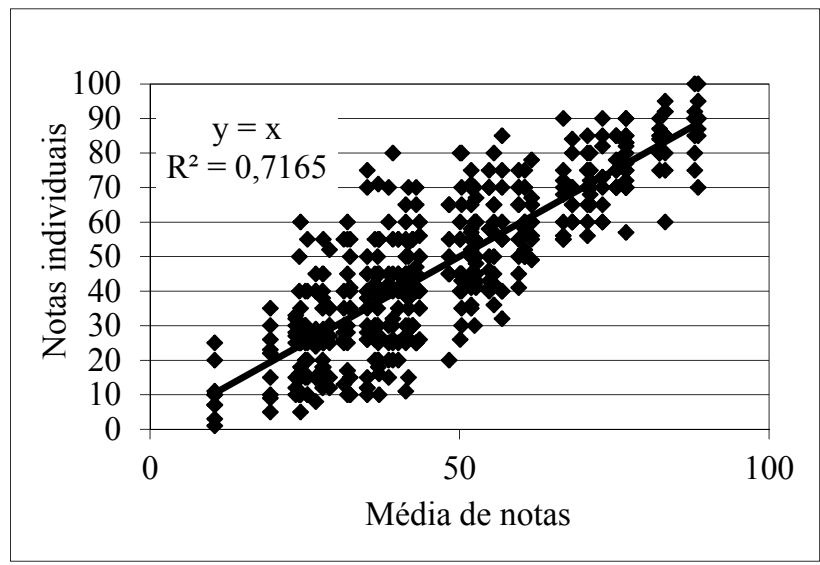

Figura 6: Correlação obtida na avaliação das 55 fotografias pelo painel treinado.

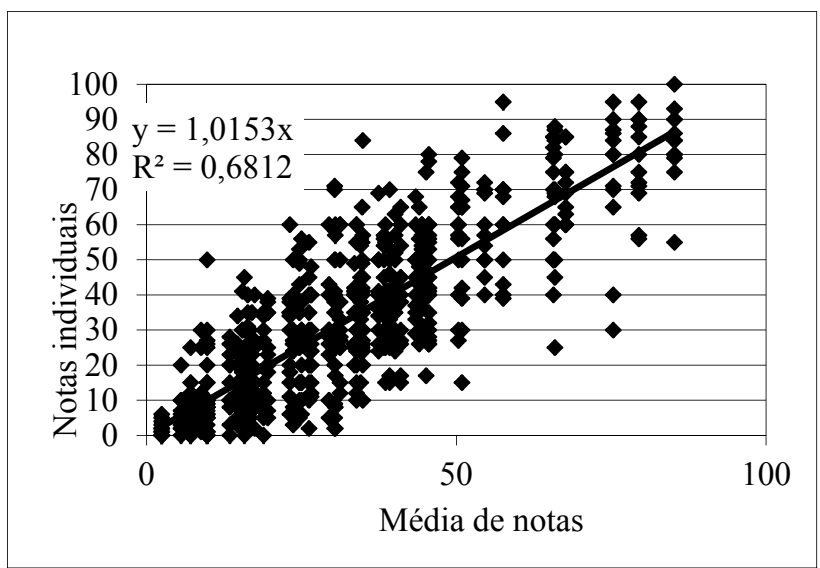

Figura 7: Correlação obtida na avaliação das 55 fotografias pelo painel não treinado.

A partir do exposto verifica-se uma correlação das avaliações do painel treinado de cerca de $84 \%$ ( $R=$ $0,84)$, enquanto que para o painel não treinado a correlação teve uma pequena diferença, sendo esta correlação de $82 \%(R=0,82)$. 


\section{CONCLUSÕES}

Nesta fase da pesquisa foi possível elaborar uma escala visual contendo fotografias de estradas de terra representativas de cada posição na escala de classificação. A medida da correlação existente entre a atribuição de notas individuais dos especialistas às fotografias de estradas não pavimentadas com a média do grupo para a montagem da escala visual foi considerada satisfatória deixando a conclusão da coerência na atribuição das classificações e, portanto, a consistência da escala visual desenvolvida.

A partir da escala e das características do rolamento relacionadas aos intervalos de notas foi possível montar o treinamento dos avaliadores para avaliar a influência exercida pelo treinamento no julgamento subjetivo da qualidade superficial das estradas. Neste ponto as principais conclusões encontradas foram que a verificação do treinamento do painel foi satisfatória, pois a partir de uma avaliação teste com cerca de 20 fotografias representando diversas condições de superfície de estradas de terra logo após o treinamento obteve-se uma correlação entre os membros de $88 \%$, ou seja, esta correlação demonstrou o nível de coerência de avaliações. $E$, além disso, verificou-se que após submeter ambos os painéis (treinado e não treinado) a avaliação de 55 fotografias obteve-se em cada painel correlações muito satisfatórias e com pouca diferença, sendo obtida para o painel treinado uma correlação de $84 \%$ e para o não treinado uma correlação de $82 \%$.

Assim conclui-se que ambas as formas de avaliação, com ou sem treinamento, podem ser utilizadas, pois a coerência oriunda da avaliação com ambos os procedimentos foram muito próximas.

Se apoiando nos comentários feitos por Fontenele e Sória (2005) sobre as prováveis causas das dispersões percebidas nos resultados de seu estudo das avaliações subjetivas de estradas não pavimentadas, verifica-se nos resultados desta etapa da pesquisa de utilização de escalas visuais no treinamento para avaliações subjetivas que as dispersões entre os avaliadores podem ser atribuídas ao fato do grande número de intervalos da escala de classificação, a certa inexperiência dos membros do painel e pouco tempo de adaptação com o processo utilizado. Para uma análise mais aprofundada consta do planejamento da pesquisa fazer o mesmo procedimento em campo e confrontar os resultados verificando se houve alguma divergência significante daqueles obtidos anteriormente.

Vale ainda ressaltar que sem dúvida uma escala de avaliação com menor número de intervalos, como por exemplo, cinco (muito ruim, ruim, regular, bom e muito bom), poderia facilitar a avaliação do painel de avaliadores, reduzindo o desvio por ventura ocasionado com a utilização de escalas de mais de cinco classes. Tal análise ainda será feita na sequencia desta pesquisa.

\section{REFERÊNCIAS BIBLIOGRÁFICAS}

CAREY, W.N; IRICK, P.E. The Pavement Serviceability Performance Concept. Highway Research Board, n. 250, p. 4058, 1960.

CHAMORRO, A.; DE SOLMINIHAC, H. E.; SALGADO, M.; BARRERA, E. Development and Validation of a Method to Evaluate Unpaved Road Condition with Objective Distress Measures. Transportation Research Record $n^{\circ} 2101$, p. 3-9, 2009.

CHAMORRO, A.; Tighe, S. Condition Performance Models for Network-Level Management of Unpaved Roads. Transportation Research Record n² 2204, p. 21-28, 2011.

DEPARTAMENTO NACIONAL DE ESTRADAS DE RODAGEM. DNER ES 128/83 - Levantamento da Condição da Superfície de Pavimento Flexíveis e Semirrígidos para Gerência de Pavimentos a Nível de Rede. Rio de Janeiro, 1983.

DEPARTAMENTO NACIONAL INFRAESTRUTURA DE TRANSPORTES. DNIT PRO 009/2003 - Avaliação Subjetiva da Superfície de Pavimentos. Rio de Janeiro, RJ, 2003a.

DNIT PRO 006/2003 - Avaliação Objetiva da Superfície de Pavimentos Flexíveis e Semi- Rígidos. Rio de Janeiro, 2003b.

DOBSON, E. F.; POSTILL, L. J. Classification of Unpaved Roads in Ontario. Transportation Research Record $n^{\circ} 898$, p. 36-46., 1983.

EATON, R. A; GERARD, S.; DATILLO, R. S. A Method for Rating Unsurfaced Roads. Transportation Research Record no 1106,v. 2, p. 34-42, 1987.

FONTENELE, H. B. Estudo para Adaptação de um Método de Classificação de Estradas Não Pavimentadas às Condições do Município De São Carlos/SP. São Carlos. 227 p. Dissertação (Mestrado) - Escola de Engenharia de São Carlos, Universidade de São Paulo, 2001.

FONTENELE, H. B; SÓRIA, M. H. A. Uma análise de avaliações subjetivas em estradas não pavimentadas. $V$ Encontro Tecnológico da Engenharia Civil e Arquitetura - ENTECA. Maringá, 2005.

GEIPOT. Anuário Estatístico dos Transportes. Disponível em: http://www.geipot.gov.br/NovaWeb/IndexAnuario.htm.

Acesso em 10 de março de 2008.

HAAS, R. et al. Modern Pavement Management. Krieger Publishing Co. Malamar, Flórida: 1994.

HARTGEN, D. T. et al. Visual Scales of Pavement Condition: Development, Validation, and Use. Transportation Research Record no 893, p. 1-6, 1982.

HUTCHINSON, B. G. Principles of Subjective Rating Scale Construction. Highway Research Record no 46, 1964. 
HUNTINGTON, G.; KSAIBATI, K. Unsealed Gravel Road Management: State of the Practice in American Counties. Transportation Research Record: Journal of the Transportation Research Board. TRB 2011 Annual Meeting CD-ROM. Washington, D. C., 2011.

JANOFF, M.; NICK, J. Effects of vehicle and driver characteristics on the subjective evaluation of road roughness. Measuring Road Roughness and Its Effects on User Cost and Confort, ASTM STP 884. T. P. Gillespie and Michael Sayers Eds., American Society for Testing and Materials, Philadelphia, p. 111-126, 1985.

JONES, J.; KSAIBATI, K. Traffic Characteristics on Unpaved Roads. Transportation Research Record: Journal of the Transportation Research Board. TRB 2012 Annual Meeting CD-ROM. Washington, D. C., 2012.

NAIR, S. K.; HUDSON, W. R. Serviceability prediction from userbased evaluations of pavement ride quality. Transportation Research Record n¹084, p. 66-75, 1986.

NAKAMURA, V. F.; MICHAEL, H. L. Serviceability ratings of highway pavements. Highway Research Record 40, p. 21-36, 1963.

NAMUR, E.; SOLMINIHAC, H. E. Roughness of Unpaved Roads: Estimation and Use as an Intervention Threshold. Transportation Research Record n²101, p. 10-16, 2009.

PEREIRA, P.; DELANNE, Y. Caracterização da Irregularidade Longitudinal dos Pavimentos Rodoviários - Definições e Especificações. Departamento de Engenharia Civil, Universidade do Minho, 1999.

SÓRIA, M. H. A.; FONTENELE, E. B. Field Evaluation of Method for Rating Unsurfaced Road Conditions. Transportation Research Record no 1819, v. 1, p. 267-272, 2003.

WEAVER, R. J.; CLARK, R. O. Psychophysical scaling of pavement serviceability. Official Insurance $n^{\circ}$ 7.41-6-SEM9/77, New York State Departament of Transportation, State campus, Albany, New York, 1977. 